\title{
InterCUBE: A Study into Merging Action and Interaction Spaces
}

\author{
Benjamin Salem $^{1}$ and Harold Peeters ${ }^{2}$ \\ ${ }^{1}$ Department of Industrial Design, Eindhoven University of Technology, The Netherlands \& \\ School of Science and Technology, Kwansei Gakuin University, Japan \\ ${ }^{2}$ Philips Medical Systems, The Netherlands \\ mail@bsalem.info, ${ }^{2}$ haroldpeeters@gmail.com
}

\begin{abstract}
We describe the development of a novel tangible interface we call the InterCUBE, a cube-shaped device with no external buttons or widgets. We study the implications of such a shape in terms of interactions, notably the degrees of freedom available and the manipulations possible. We also explain and investigate the merging of the action, perception and interaction spaces, and we design the InterCUBE interaction accordingly. To investigate the system we have implemented a demonstration application: a shopping menu, in which users can navigate through a menu simply by turning the cube in either one of the four possible directions. We have evaluated the InterCUBE in comparison to an equivalent mouse based interface and discuss the results.
\end{abstract}

Keywords. Tangible User Interface, Usability, action and interaction spaces.

\section{Introduction}

We have developed the InterCUBE, a cube-shaped TUI (Tangible User Interface) with inherent added value. We have also integrated the action space with the interaction space. Whereby the InterCUBE is both the input and the output device. IN doing so we hope that the user attention focus will be kept consistent during the interaction. We advocate that while acting on the device, reacting to the device and interacting with an application, the user focus should be kept as much as possible onto one single point. This is a different approach to other cube-shaped THUI. In comparison computer graphics projection systems such as ToolStone [1] are different because there is a distinction between the TUI as input device and the output device that is a separate display. So are other cube-based systems such as the Cognitive Cubes [2], in this instance they do not allow for output channels. Although at this stage of development, the only output we have implemented on the InterCUBE is an LED on each face of the cube that indicates which side is up (and currently active). Intended developments include addition of displays on each side of the cube to visualise a menu.

\subsection{Tangible Computing}

Tangible computing generally refers to computing systems that use physical artifacts as representations and controls for digital information [3]. In other words, tangible 
computing is about having devices that represent some information by ways of their colour, behaviour, sound or other properties. In the spectrum of interaction from digital to physical tangible computing lies at the physical end. At one extreme are virtual reality and Graphical User Interfaces. Their main focus is the interaction within the digital context (e.g. Virtual Environment). Then come ubiquitous computing, augmented reality (also known as mixed reality) and ambient intelligence. They include interactions that are physically contextualized, e.g. attached to some physical objects. At the other end of the spectrum, tangible computing is mainly concerned with physically embodied interaction. Tangible devices can offer the benefits of strongly integrated physical representations of digital information. This is because the hands and the brain have a particularly strong partnership [4]. We have undertaken the development of a device that complies with this TUI vision but is also a relevant object as well. An object with intrinsic aesthetic value and intuitive to use.

\section{Action, Perception and Interaction Spaces}

In terms of the immediately surrounding physical world, we rely on our hands for exploring, manipulating and reshaping it. From cognition perspective the hand has two main purposes: (1) the manipulation (i.e. action) and (2) the experience (i.e. perception) of objects. When manipulating objects in the real world, action (hands and fingers) and perception (the object in the real world) coincide in time and space [5]. The merger of action and perception spaces is intrinsic to TUI. Users have to grasp and manipulate (actions) the TUI and experience haptics (touch and weight) and motor experience (perception) of the device.

It also has been proven that offering task-relevant information in the same space as where actions take place leads to increased performance [6]. This suggests a merging of the user interface (task relevant information) and the device (where action take place). In other words a merging of (1) the action, (2) the perception and (3) the interaction spaces. This merger would ensure that the user attention is at the same time on what s/he is doing, where feedback will be experienced and where the application interface is displayed to her/him. All these happen to be on the same point. In comparison, with a screen-keyboard-mouse user interface, there is a separation between these spaces, given by the physical separation of input and output devices.

\subsection{Affordance}

The affordance of a form can be described as action priming activated by the appearance of an object. Although mostly visual the InterCUBE, other appearances are also relevant, such as weight and texture. The appearance of an object is triggering the user into anticipating and planning possible actions towards that object. These actions are related to the manipulation and perception of the object, for example a grasping action on a small object. At the initiation of such a grasping action people pre-shape their hands in order to match hand and object shapes [8]. We have, thus, facilitated such a pre-shaping by using a known basic form and by ensuring that the dimensions of the cube match those of the hand palm of an adult user. These ideas 
stem from the concept of affordance, first suggested by [9] and later applied to design by [10]. Designing a cube shaped TUI has therefore two advantages: (1) the cognitive load related to the manipulation of the TUI is kept to a minimum thanks to the clear affordance of the cube; and (2) the pre-shaping of the hand is facilitated thanks to the familiarity users have with the cube form. We find it important and relevant that interactive products clearly indicate the kind of interaction they are meant to support, this can be achieved thanks to the clear affordance of their form.

When an object affordance has been recognised, an attention grabbing effect occurs [7]. Thus if we have reinforce the affordance and simplify the manipulation planning of a TUI, we would facilitate the attention grabbing effect. To ensure the most efficient attention grabbing, such grabbing would have to be short and to the point. Short in time duration and to the point in term of relevance of the manipulation afforded. With an efficient attention grabbing, the TUI should leave more time for the user to perform other cognitive tasks. Liberate the user from having to think too long about how to grasp, manipulate and use the TUI presented. In the current implementation of the InterCUBE we have tried to address the issue of action and perception spaces merger. We have developed a device where there is no pre-requisite in terms of hand positions or handling. The simple affordance and the straightforward manipulations available, reduce greatly the complexity of interaction at the level of the device. This helps the user to focus on the menu interaction currently displayed on a monitor, instead of first paying attention to the manipulation of the InterCUBE and then observing the effects of his actions on the menu. The user focus is kept on to the same point, i.e. the GUI, and subject to a minimum of change.

\section{Designing the InterCUBE}

From a product design point of view our approach was about coming up with shapes that delivered simple and easy to use objects. Hence the development of our TUIs based on the exploration of pure aesthetic forms. We have investigated various physical forms that could be suitable for a TUI and have focused on basic ones, such as cube, sphere, and pyramid. Such forms are familiar to everyone. With a basic form it is possible to avoid any distraction due to the exploration of the form if it were complex or novel. Furthermore, having selected the form to be used, the implementation of a TUI was based on the understanding of what affordance such a form would have and how to implement them in an interface. This approach has led in the first instance to the development of the InterCUBE a cube shaped device, as the implementation of these principles.

\subsection{Cube Based TUIs}

While designing a cube shaped device, we were inspired by the early work on multidimensional icons, developed as a cubic shape in replacement of the widely used 2D icons [11]. Since then, there has been a substantial developments in cubic shaped TUIs. Solutions spanned from single [12] to modular devices [2], and from rigid [13] to deformable shape [14]. The interaction principles used were covering a wide spectrum of possibilities, from manipulation to composition. Manipulations were 
generally about rotating and positioning the TUIs. An early example is Bricks, as physical handles to manipulate digital objects [15]. The bricks were lying on a horizontal screen and the rotation and displacement on that horizontal plan were translated into an interaction. Within the same principle, ToolStone was developed with further investigation of the manipulations allowed [1]. ToolStone could be rotated, flipped over, tilted and moved in 3D. A Playful interface for home entertainment system based on a cube is another example of such TUI [16]. In this instance it is the rotation of the cube that is implemented in the interface. This aspect is similar to our development of the InterCUBE.

At the other end of the spectrum compositions are about assembling various TUIs into a functional grouping. This approach was adopted with ActiveCube, for instance it is the 3D arrangement of cubes that allow the construction and interaction with a $3 \mathrm{D}$ environment [17]. Navigation Blocks is another implementation. It is a system based on several blocks that orientation, movement and relative position correspond to various database queries [13]. Other examples ones include cognitive cubes [2] and mediaBlocks [18]. In comparison, to these TUIs, the InterCUBE is a stand-alone device.

Most of the TUIs based on the manipulation principle are projects involving a cube shaped TUI as a replacement for some functions of the mouse as an input device, like the cubic mouse. It is used to orient graphic models, joysticks on each of the cube faces are used for operation on each of the $\mathrm{x}, \mathrm{y}$, and $\mathrm{z}$ axis [12]). Another instance of such TUI is the Cubik, developed for the creation and manipulation of 3D models. It is based on push and pull widgets [19]. However, it is not necessary to rely on a rigid device like the InterCUBE. Indeed, a deformable cube was developed as a direct input device for the deformation and thus manipulation of a 3D shape [20].

Table 1. Various Cube based Devices

\begin{tabular}{|c|c|c|c|c|}
\hline Device & Type & DoF & Interaction principle & Ref. \\
\hline Cubic Mouse & Device & 6 & $\begin{array}{l}\text { Manipulate Joysticks and buttons on each } \\
\text { face of cube }\end{array}$ & {$[12]$} \\
\hline Toolstone & Token & 6 & $\begin{array}{l}\text { Rotations and Translations but must keep } \\
\text { contact with base }\end{array}$ & {$[16]$} \\
\hline Brick & Tokens & 4 & Rotations and Translations & {$[15]$} \\
\hline Cubik & Device & 3 & $\begin{array}{l}\text { Push and Pull of the cube (decrease and } \\
\text { increase of size) }\end{array}$ & [19] \\
\hline Tangible Cube & Device & 3 & Rotations & {$[16]$} \\
\hline ActiveCube & Modules & 3 & $\begin{array}{l}\text { Arrangement of cubes together, yielding } \\
\text { different structures }\end{array}$ & {$[17]$} \\
\hline Cognitive Cube & Modules & $\mathrm{m} 3$ & Same as ActiveCube & [2] \\
\hline $\begin{array}{l}\text { Navigational } \\
\text { Blocks }\end{array}$ & Token & 3 & Arrangement of cubes together & {$[13]$} \\
\hline Mouse & Device & 3 & $\begin{array}{l}\text { Translation on a horizontal plan ( } 2 \mathrm{DoF}) \\
\text { and a scroll wheel ( } 1 \mathrm{DoF}) \text {. }\end{array}$ & \\
\hline InterCUBE & Device & 2 & Rotations & \\
\hline MediaBlocks & Tokens & $\mathrm{n} / \mathrm{a}$ & $\begin{array}{l}\text { Insertion or removal of blocks from a } \\
\text { console }\end{array}$ & {$[18]$} \\
\hline DO-IT & Device & $\mathrm{n} / \mathrm{a}$ & Deformation of the cube. & {$[20]$} \\
\hline
\end{tabular}


It is noticeable that few projects go as far as developing the TUI as a physical representation of some digital information. An exception is ActiveCube with an LED array display that represents some information from the application [21]. This is the closest to the concept we are thinking of when advocating the merger of the action and the interaction spaces (as described in section 4.1).

It is also remarkable that no comparison between the various devices proposed and the mouse has been included in the above papers. One would have hoped that if any of the devices was to become as widely used as the mouse for an application or an interface, there should be some comparison made. In table 1 (next) we list the cubebased interfaces and compare their DoF (Degree of Freedom), we also include the mouse.

As comparison, in the case of the InterCUBE we have ensured that there are no buttons, widgets or other features that could invite manipulations. The only possible manipulations remain holding, moving and rotating the cube.

\section{Interaction Design}

The InterCUBE is an unconstrained token that is hand held. It can be rotated and moved freely with no physical constraints. As such it is a device can have up to 6DoF. This is a substantial extension of possibilities compared to cube shaped token combined with a device (e.g. MediaBlocks). Such token system falls under the Token+Constraint classification [22]. In this classification the TUI is based on a token and a reference device (such as an active surface) that reduce the available DoF of the token. From this perspective.

Within the current implementation, we have limited the DoF implemented as part of the interface to : rotations forth/back and rotations right/left. During these rotations, the very nature of the cube shape yields a device with two states, discreet and transitional. The discreet states correspond to one of the face facing upwards towards the user and the transitional states correspond to the rotation from one orientation/face to another (in other words from one discreet state to another). The current state is then used as a departure point for the selection of the next option. Before reaching the desired option the device goes through a transitional state when the cube is being rotated, and no face is fully upwards. There is a threshold at midway between two discreet states when the interface is shifted from the previous state/option to the next. By design, this happens at around 45degrees of the rotation. In the current version, where the device is used for menu navigation, at the 45 degrees angle the user can see that the menu changes to the next options.

\subsection{User Actions and User Interaction}

The only actions necessary to interact with the cube are picking it up and turning it either up, down, right or left. There are many implications from the user interface (UI) point of view. The InterCUBE could be used for object manipulations or menu navigation. If the device is to be used for $2 \mathrm{D}$ or $3 \mathrm{D}$ objects manipulations, there could be a direct mapping between the user actions on the InterCUBE and their translation into manipulations of an object. Such a mapping could be done in two ways. The 
InterCUBE being a discreet device, the mapping could be either (1) discreet to discreet or (2) discreet to continuous. For the object manipulation interface to work the rotation of the InterCUBE could be (1) a rotational step the object should follow, of which the ration device/object rotations could be controlled or (2) an indication of which direction the object should rotate towards, and the rotational speed could be specified by the angle of rotation of the device.

In the case of menu interaction, the InterCUBE can be mapped to a menu structure that takes into account the 4 possible choices available at each step (corresponding to the 4 possible faces that can be turned up). This a series of discreet states, for the interface to work, we need a discreet to discreet mapping and a one to one mapping between rotation and menu steps. The menu structure will hence be made of a series of decision tress with 4 branches at each node.

Within the developed system we have implemented a shopping application. We have selected this application as it fits well with the requirements of a menu structure. Users can browse through some clothes shopping categories (starting at Women, Kids and Men) and down to clothing items such as trousers, ties, pyjamas... At each step of the interaction the user is faced with a maximum of 4 possible options.

\section{Comparing the InterCUBE and the Mouse}

In our opinion this is relevant and important to compare the InterCUBE to the mouse as we are hopping our device could emerge as replacement or as complement to the mouse in some specific applications. In particular applications where efficiency is not that relevant compared to for example the experience. In its current implementation the cube is designed for menu based interactions. It therefore could be compared with an equivalent setting where the mouse is used. Even though there is a difference in $\mathrm{DoF}$ and handling principles between the two devices. Bearing in mind that computer users are by now all familiar with the mouse, it puts the InterCUBE in an unfavorable starting position. Nevertheless, in the next parts of this paper we present and discuss some comparative experiments, between the InterCUBE and the Mouse.

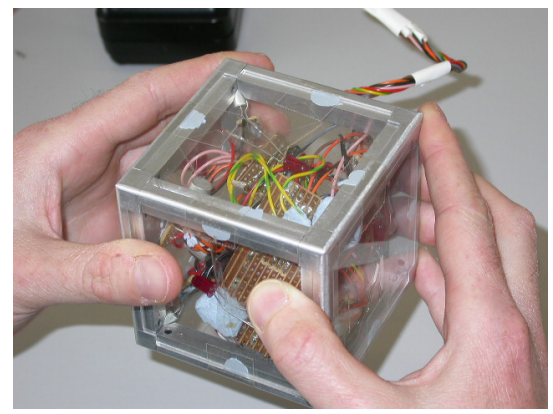

Fig. 1. The InterCUBE 


\subsection{Tests to Evaluate the InterCUBE}

The comparison tests were set up whereby users had to perform the same tasks using one and then the other device. The users had to complete all the tasks using one device before moving onto using the other device. The one condition changed was the system configuration: Mouse or InterCUBE based. The device order was randomly chosen with half participants starting with the InterCUBE and half with the mouse. This would eliminate the possible effects of mutual influence of devices. We ran a series of assessments: (1) subjective assessment and, (2) objective assessments. In the subjective assessment, we would like to evaluate how intuitive the InterCUBE, a novel input device is. Would the test subjects be able to use the InterCUBE at all? . We also assessed the perceived usability of the InterCUBE when judged directly and the perceived usability of each device when compared one against another. This was done through a questionnaire. In the objective assessment the InterCUBE efficiency was compared to the mouse. In this assessment equivalent time measurements were compared between the two devices for a series of tasks.

In our tests the independent variables were the Menu and GUI, Device used and the dependent variables were the tasks performance.

\subsection{Participants}

Students of the Department of Industrial Design were the test participants. All of our students have significant computer and Internet experience. Most of the students were in the age group 18 -25 years. As they are ID students they will be more open in using alternative technological systems in performing a task. At least 20 (N22) individuals took part in the test.

\subsection{Methods and Scenarios}

We have run a series of comparative studies between the InterCUBE interface and a mouse interface. Participants were given a list of tasks to perform arranged in three major parts for each device (as described in table 2). Our aim is to establish a comparative study between the mouse and the InterCUBE based on the performance of a series of tasks of increasing complexity, thus increasing the cognitive load on the user: (1) single manipulation tasks without a Graphical User Interface (GUI), (2) sequence of manipulations without GUI and finally (3) full realistic shopping tasks with GUI. So we could compare the resulting time measurements between single manipulations, then between sequences of manipulations and finally between a fully fledge GUI setting. Furthermore we also investigate the ratios of time duration between manipulation sequences without and with a GUI (i.e. equivalent tasks with increased cognitive load on the user).

We perform a measurement of the time duration of each task for quantitative analysis (see table 2 for description of tasks), as well as a post-test questionnaire for qualitative analysis. All tests were recorded with a video camera and the subjects knew this. This was to ensure we captured detailed information in case we noticed some unusual data or comments from the participants. 
Table 2. List of tasks. The performed tasks were in random order within one part, however the parts order was not randomised as it relate to a cognitive load build-up. The order of which device to use was also random. In the case of the mouse the users were not required to bring the mouse back to the initial position after each movement. Tasks of part 2 and 3 are identical but without and with the Graphical User Interface.

\begin{tabular}{|c|c|}
\hline InterCUBE & Mouse \\
\hline \multicolumn{2}{|c|}{$\begin{array}{l}\text { On both columns the tasks are identical but translated into InterCUBE or mouse actions } \\
\text { Part } 1 \text { : Single step manipulation }\end{array}$} \\
\hline $\begin{array}{l}\text { Turn the cube right } \\
\text { Turn the cube left } \\
\text { Turn the cube forwards/up } \\
\text { Turn the cube backwards/down }\end{array}$ & $\begin{array}{r}\text { Move the mouse right } \\
\text { Move the mouse left } \\
\text { Move the mouse forwards/up } \\
\text { Move the mouse backwards/down }\end{array}$ \\
\hline \multicolumn{2}{|c|}{ Part 2 : Manipulation without Graphical User Interface } \\
\hline $\begin{array}{l}\text { Turn the cube: } \\
\text { down-down-down-left-down-down-any side } \\
\text { Turn the cube: } \\
\text { down-left-left-left-down-down-any side } \\
\text { Turn the cube: } \\
\text { down-right-right-right-down-down-down- } \\
\text { any side }\end{array}$ & $\begin{array}{r}\text { Move the mouse: } \\
\text { down-down-down-left-down-down-any } \\
\text { direction } \\
\text { Move the mouse: } \\
\text { down-left-left-left-down-down-any } \\
\text { direction } \\
\text { Move the mouse: } \\
\text { down-right-right-right-down-down-down- } \\
\text { any direction }\end{array}$ \\
\hline \multicolumn{2}{|c|}{ Part 3 : realistic shopping tasks with menu } \\
\hline $\begin{array}{l}\text { Buy the "K } \\
\text { Buy the "W } \\
\text { Buy the" }\end{array}$ & $\begin{array}{l}\text { ids pyjama } 1 " \\
\text { omen shirt } 1 " \\
\text { "Men tie } 2 "\end{array}$ \\
\hline
\end{tabular}

An experiment pack was distributed to all the participants. It included a questionnaire and some introductions and short explanations about the experiment and the devices used. Subjects were asked to fill a questionnaire consisting of four parts: (1) Participant background, to know and understand the type of user and the relevance of her/his comments (this part filled before the tests); (2) Assessment of the InterCUBE setting, to find out the perceived usability of the InterCUBE within the setting of the experiment; (3) Assessment of the Mouse setting, to find out the perceived usability of the mouse within the setting of the experiment; ( 2 and 3 were swapped in case of different device order); (4) Relative ranking by the user of the InterCUBE Vs the mouse devices, to discover the user perceived preference.

\subsection{Experiment Setting}

The experiment was set-up using two different computer systems one based on the InterCUBE and the other on the mouse. However both systems run the same application (i.e. a shopping menu) and user interface as far as possible (i.e. layout, levels and options etc). Both settings were in an office environment and using 
common arrangements as much as possible. That is the displays are on a desktop, the devices are positioned in front of the displays and the users are required to sit right in front of one of these arrangements at a time. One key difference however, is that for the InterCUBE system the display is actually on a horizontal plane, parallel to the top surface of the cube. This is to simulate the intended display on the topside of the InterCUBE. As for the mouse system we kept to the common settings of a desktop PC.

While the InterCUBE selection is done by rotating the device the mouse selection is done by moving the device over the selected option and clicking the mouse left button. To allow for a fair comparison, we have set the distance between the options in the mouse system to be such that the time for mouse displacement should be approximately the same than an average rotation of the InterCUBE. Within the menu structure, options have consistent and identical positions on the display. The option at the top of the display is always used to go back, and the option at the bottom, when applicable, is used as a confirmation of choice. Left and Right options are used for choices, and when not available are simply not displayed. Both menus are identical and have a maximum of four options located at the top, bottom, right and left of the display.

\section{Evaluation Results}

\subsection{Subjective Study}

The participants were required to subjectively assess both the InterCUBE and the Mouse. This was done using a satisfaction questionnaire, the System Usability Scale (SUS) (Brooke, 1986). This is a bipolar rated questionnaire. The users have answered that for both they feel positive (SUS scores above 50\%) without significant difference between the two devices. There is no significant difference between the perceived usability scoring of the InterCUBE (mean 77.8, std 2.3, N 22) and of the mouse (mean 79.9, std 3.5, N 22), (ANOVA, $\mathrm{df}=1, \mathrm{~F}=0.167, \mathrm{p}=0.687$ ). Within the context of evaluation and application, the InterCUBE was therefore assessed as being as good, in perceived usability, as the mouse.

As part of the subjective study, users were asked four questions about the two devices. When asked the three questions next, there was no significant advantage for either the mouse or the InterCUBE (bipolar aggregate scoring with 1 for mouse and 22 for InterCUBE a score of 66 would be neutral): (1) I felt more in control using the mouse or the InterCUBE (mean 2.86, N 22). (2) I felt more exhausted after using the mouse or the InterCUBE and (mean 3.04, N 22). (3) Performing the tasks was easier while using the mouse or the InterCUBE. (mean 3.31, N 22). However there was a significant advantage for the InterCUBE with the following fourth question: I prefer the interface together with the mouse or the InterCUBE (mean 3.90, N 22). These results indicate that there is no overwhelming difference between the two devices, however there is a subjective preference for the InterCUBE. Even though none of the participants had prior experience with the device. 


\subsection{Objective Study (Time Measurements)}

While performing the tasks described in table 4, the time duration of the InterCUBE and the mouse interaction were measured in seconds. Figure 7 shows the results of these measurements.

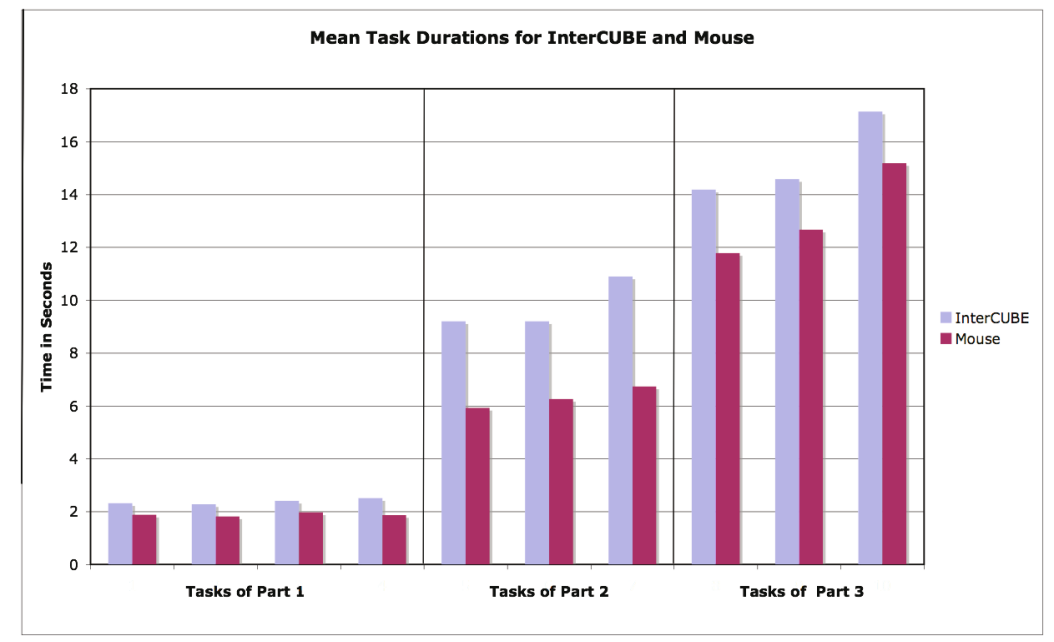

Fig. 2. Tasks duration as measured in seconds for both the InterCUBE and the mouse. There is a significant difference between the two sets of measurements. The mouse is significant faster for all the individual tasks with a mean difference of $\sim 2 \mathrm{sec}$. Note that the perceived increase trend between tasks is to be ignored as the data represent different tasks of increased cognitive load. Part 1: single step manipulation, part 2: manipulations without GUI, part 3: manipulations with GUI.

The mouse yields significantly faster results than the InterCUBE, for all the tasks performed. The mean difference is approximately 2 seconds. Furthermore, we have also investigated the comparison of time duration of parts 2 and 3 of the test (i.e. without and with a GUI). Such a comparison for both the InterCUBE and the mouse gives interesting results. The ratio for both sets of data is calculated by dividing the duration of task performance with GUI by the time duration of task performance without GUI. We compare the ratio of the InterCUBE with the ratio of the Mouse and we have discovered that it is significantly smaller (Sig. 0.004) for the InterCUBE data than it is for the mouse data. In other words, the addition of an interface yields a relative larger increase of the duration of a mouse based task compared to an InterCUBE based task. This implies that within the current context of evaluation and application, the cognitive load of the GUI is smaller with the InterCUBE than it is with the Mouse. Indeed the impact of the interface is a third longer with the mouse than with the InterCUBE (1.33 average ratio, see figure 4). This indicates that if the action space and the perception space are merged, there is significantly less of a cognitive load on the user than if the two spaces are separated. In the case of the InterCUBE, the user manipulates the device and gets haptics and motor feedbacks 
from it. The user attention can focus on the display and the menu interface. In the case of the mouse, the user manipulates the device but obtain feedback on the separate display, where s/he needs to check that he is moving the mouse pointer onto the desired option.

In our experiment, the Mouse performs better than the InterCUBE. However, it would be useful to investigate if this implies that further training would result in the InterCUBE being significantly better than the mouse within the context of specific applications. In particular with the planned integration of displays on the device that will result in a device with merged action, perception and interaction spaces.

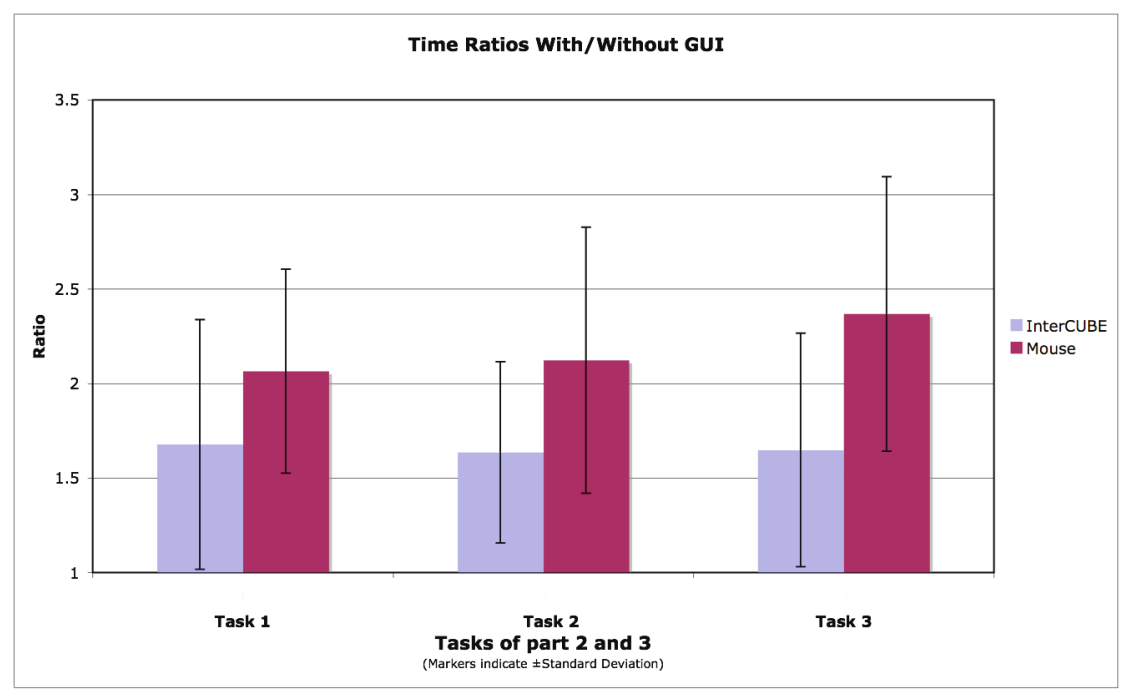

Fig. 4 . The time ratio for both devices according the three tasks performed. The InterCUBE ratio is significantly smaller that the mouse one.

\subsection{Further Results}

During the experiment, it appeared that $45 \%$ of users have suggested a smaller size for the InterCUBE. Users made such a comment in the hope that the device could be easily manipulated by one hand only. Interestingly enough, this was a fact that was overseen during the development of the InterCUBE. The device was designed to fit on one hand but must have been oversized. Another clear request from the users (77\%) is for the removal of the umbilical cord used for power supplied and signal transmission. This should be addressed in the next developments.

\section{Conclusion}

Through this paper we have presented the current developments of the InterCUBE as a device for menu navigation. We have selected a shopping menu application for 
demonstration and evaluation. Rather than just investigate the device we have compared its performances with those of a mouse in an equivalent setting. With hindsight, we realise that the training and familiarity users have with the mouse make it a formidable challenger. However our tests have shown that the InterCUBE when used for the first time is still an acceptable contender. The different tests seem to be enough training to let the user handle the InterCUBE sufficiently well to finish all the tasks. Significant training on the InterCUBE would probably yields more balanced results. Our results show that a novel, unknown interaction device could be used as a replacement to the mouse within specific context. In such a context, the InterCUBE was favoured due to it being perceived as easier to use than the mouse. Even in a case like ours where users have no prior training. The single manipulation and the sequence manipulation without interface were expected to be sufficient training, but probably not to balance out the effects of five years experience with the mouse. It would be interesting to run similar tests with users unfamiliar with either device. Summary explanations given in the experiment packs to users and the part 1 of the experiments are enough for them to figure out how to use the InterCUBE. This has opened a promising perspective, one of the development of a series of novel TUI based on our interest with basic pure forms and common action space, perception and interaction space like in old fashion tools. Our objectives are two folds: (1) render the InterCUBE a device that does merge the action, perception and interaction spaces, beyond the limited LED currently used to display the active face and (2) deliver a stand-alone device with processing units, power supply and application all integrated within the cube. Our future plans call for the integration of LCD displays on each side of the device. As well as for the integration of the running application in an on-board circuit. Additionally such stand-alone device would avoid movement hampering and cable entanglement due to the current umbilical cord. We would like to test the system with users who have no mouse experience or with reduced motor skills (young children, elderly). We are also interested in other applications such as gaming.

Acknowledgments. The InterCUBE was initially developed by the first author while at Polywork Ltd. The original idea of the InterCUBE comes from a cooperation with Alex Watkins, an artist interested in boxes. We also wish to thank the following people for their help and advices : Ben Patterson, Rob Yates, Lorenzo Cervera and Matthias Rauterberg .

\section{References}

1. Rekimoto, J., Sciammarella, E.: ToolStone: Effective Use of the Physical Manipulation Vocabularies of Input Devices. In: Proceedings of UIST 2000, pp. 109-117. ACM, New York (2000)

2. Sharlin, E., Itoh, Y., Watson, B., Kitamura, Y., Sutphen, S., Liu, L.: Cognitive Cubes: A Tangible User Interface for Cognitive Assessment. In: Proceedings of CHI 2002, New York, NY, USA, pp. 347-354. ACM, New York (2002) 
3. Dourish, P.: Where the action is: The foundation of embodied interaction. MIT-Press, Cambridge, Massachusetts (2001)

4. Flanagan, J.R., Johansson, R.S.: Hand Movements. In: Encyclopaedia of the Human Brain, vol. 2, Elsevier Science, Amsterdam (2002)

5. Rauterberg, M.: Ueber die Quantifizierung software-ergonomischer Richtlinien, PhD Thesis. University of Zurich. Refered in Fjeld, M., Bichsel, M., Rauterberg, M.: BUILDIT: a brick-based tool for direct interaction. In: Harris, D.(ed.) Engineering, Psychology and Cognitive Ergonomics, vol. 4, pp. 205-212, Ashgate, Hampshire (1995)

6. Hacker, W., Clauss, A.: Kognitive Operationen, inneres Modell und Leistung bei einer Motagetatigkeit. Deutscher Verlag des Wissenschaften, Berlin, Germany. Refered in Fjeld, M., Bichsel, M., Rauterberg, M.: BUILD-IT: a brick-based tool for direct interaction. In: Harris, D. (ed.) Engineering, Psychology and Cognitive Ergonomics, vol. 4, pp. 205-212, Ashgate, Hampshire (1999) (1976)

7. Handy, T.C., Grafton, S.T., Shroff, N.M., Ketay, S., Gazzaniga, M.S.: Graspable object grab attention when the potential for action is recognised. Nature Neuroscience 6(4), 421$427(2003)$

8. Schettino, L.F., Adamovich, S.V., Poizner, H.: Effects of object shape and visual feedback on hand configuration during grasping. Experimental Brain Research 151, 158-166 (2003)

9. Gibson, J.J.: The ecological approach to visual perception. Lawrence Erlbaum, London, England (1986)

10. Norman, D.A.: The Design of Everyday Things. MIT Press, London, England (2001)

11. Henry, T.R., Hudson, S.E.: Multidimensional Icons. ACM Transactions on Graphics 9(1), 133-137 (1990)

12. Frohlich, B., Plate, J.: The Cubic Mouse: A New Device for Three-Dimensional Input. In: Turner, T., Szwillus, G., Czerwinski, M., Paterno, F. (eds.) Proceedings of CHI2000, pp. 526-531. ACM, New York (2000)

13. Camatara, K., Do, E.Y.-L., Johnson, B.R., Gross, M.D.: Navigational Blocks: Navigating Information Space with Tangible Media. In: Gil, Y., Leake, D.B. (eds.) Proceedings of UIU-02, International Conference on Intelligent User Interfaces, pp. 31-38. ACM, New York (2002)

14. Murakami, T., Hayashi, K., Oikawa, K., Nakajima, N.: DO-IT: Deformable Objects as Input Tools. In: Proceedings of CHI95, pp. 87-88. ACM, New York (1995)

15. Fitzmaurice, G.W., Ishii, H., Buxton, W.A.S.: Bricks: Laying the foundations for graspable user interfaces. In: Proceedings of SIGCHI '95, pp. 442-449. ACM, New York (1995)

16. Block, F., Schmidt, A., Villar, N., Gellersen, H.W.: Towards a Playful User Interface for Home Entertainment Systems. In: Markopoulos, P., Eggen, B., Aarts, E., Crowley, J.L. (eds.) EUSAI 2004. LNCS, vol. 3295, pp. 207-217. Springer, Heidelberg (2004)

17. Kitamura, Y., Itoh, Y., Kishino, F.: Real-time 3D Interaction with ActiveCube. In: Jacko, J.A., Sears, A., Beudouin-Lafon, M., Jacob, R.J.K. (eds.) Proceedings of CHI2001, pp. 355-356. ACM, New York (2001)

18. Ullmer, B., Ishii, H.: MediaBlocks: Physical Containers, Transports, and Controls for Online Media. In: Proceedings of SIGGRAPH 98, pp. 379-386. ACM, New York (1998)

19. Lertsithichai, S., Seegmiller, M.: CUBIK: A Bi-Directional Tangible Modeling Interface. In: Proceedings of SIGCHI 2002, pp. 756-757. ACM, New York (2002)

20. Murakami, T., Nakajima, N.: Direct and Intuitive Input Device for 3-D Shape Deformation. In: Adelson, B., Dumais, S., Olson, J. (eds.) Proceedings of CHI'94, pp. 465-470. ACM, New York (1994) 
21. Watanabe, R., Itoh, Y., Asai, M., Kitamura, Y., Kishino, F., Kikuchi, H.: The Soul of ActiveCube - Implementing a Flexible, Multimodal, Three-Dimensional Spatial Tangible Interface. ACM Computers in Entertainment 2(4), 1-13 (2004)

22. Ullmer, B.: Tangible interface for manipulating aggregates if digital information, doctoral dissertation, MA, USA. MIT, Cambridge (2002), available at http://alumni.media.mit.edu/ ullmer/dissertation/ 